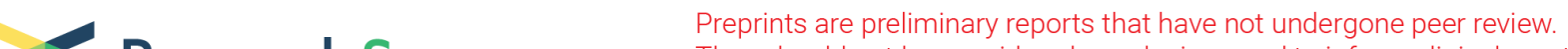 Research Square
Thr ef should not be considered conclusive, used to inform clinical practice,
of the media as validated information.
}

\section{Effectiveness of empirical anti-pseudomonal antibiotics in patients with recurrent COPD exacerbation: A multicenter retrospective cohort study}

\section{Akihiro Shiroshita ( $\square$ akihirokun8@gmail.com )}

Department of Respiratory Medicine, Ichinomiyanishi Hospital https://orcid.org/0000-0003-0262-459X

\section{Chisato Miyakoshi}

Department of Research Support, Center for Clinical Research and Innovation, Kobe City Medical Center General Hospital

\section{Shunta Tsutsumi}

General Medicine, Awa Regional Medical Center

Hiroshi Shiba

Post Graduate Education Center, Kameda Medical Center

\section{Chigusa Shirakawa}

Department of Respiratory Medicine, Kobe City Medical Center General Hospital

\section{Kenya Sato}

Department of Thoracic Medicine, Saiseikai Yokohamashi Tobu Hospital

\section{Shinya Matsushita}

Department of Thoracic Medicine, Saiseikai Yokohamashi Tobu Hospital

\section{Yuya Kimura}

Center for Pulmonary Diseases, Department of Respiratory Medicine, National Hospital Organization

Tokyo National Hospital

\section{Keisuke Tomii}

Department of Respiratory Medicine, Kobe City Medical Center General Hospital

\section{Masahiro Ohgiya}

Center for Pulmonary Diseases, Department of Respiratory Medicine, National Hospital Organization Tokyo National Hospital

\section{Yuki Kataoka}

Department of Respiratory Medicine, Hyogo Prefectural Amagasaki General Medical Center

\section{Research Article}

Keywords: chronic obstructive pulmonary disease, antibiotics, Pseudomonas aeruginosa, recurrent 
Posted Date: April 14th, 2021

DOl: https://doi.org/10.21203/rs.3.rs-418138/v1

License: (c) (i) This work is licensed under a Creative Commons Attribution 4.0 International License. Read Full License 


\section{Abstract}

Background: Although frequent chronic obstructive pulmonary disease (COPD) exacerbation has been associated with the isolation of Pseudomonas aeruginosa (PA) in sputum cultures, it remains unknown whether the empirical use of anti-pseudomonal antibiotics can improve outcomes in patients with frequent COPD exacerbations.

Research Question: Does empirical use of anti-pseudomonal antibiotics improve the length of the hospital stay in patients with recurrent COPD exacerbation?

Study Design and Methods: This multicenter retrospective cohort study was conducted in seven hospitals in Japan. We included patients aged $\geq 40$ years who were admitted to the hospital with COPD exacerbation more than twice during the study period (April 1, 2008 to July 31, 2020). The primary outcome was the length of the hospital stay. For statistical analysis, a log-linked Gamma model was used. Parameters were estimated using a generalized estimating equation model with an exchangeable correlation structure accounting for repeated observations from a single patient. Covariates included age, body mass index, home oxygen therapy use, respiratory rate, heart rate, oxygen use on admission, mental status, systemic steroid use, activities of daily living, and number of recurrences. Hospital-specific effects were specified as fixed effects.

Results: Among 1573 patients with COPD exacerbation, 344 patients and 965 observations of recurrent COPD exacerbations were selected. Anti-pseudomonal antibiotics were used in 173 patients (18\%). The estimated change in the length of the hospital stay between the anti-pseudomonal and non-antipseudomonal antibiotics groups was 0.044 days [ $95 \%$ confidence interval; $-0.077,0.166]$.

Interpretation: Use of empirical anti-pseudomonal antibiotics based only on recurrence may not be necessary. Further large-scale studies are needed to more precisely evaluate the effectiveness of empirical anti-pseudomonal antibiotics.

\section{Summary}

\section{Take-Home Point}

Study Question: Does the use of empirical anti-pseudomonal antibiotics improve the length of the hospital stay in patients with recurrent COPD exacerbation?

Results: The estimated change in the length of the hospital stay between the anti-pseudomonal and nonanti-pseudomonal antibiotics groups was 0.044 days [95\% confidence interval; $-0.077,0.166]$.

Interpretation: Use of empirical anti-pseudomonal antibiotics based only on recurrence may not be necessary.

\section{Introduction}


Chronic obstructive pulmonary disease (COPD) is one of the most common respiratory diseases. ${ }^{1}$ Patients with COPD frequently experience acute exacerbations; the event rate has been reported as approximately 1-2 times per year. ${ }^{2}$ Following COPD exacerbations, background factors such as body mass index, obstruction, dyspnea, and exercise capacity may worsen. ${ }^{3}$ Moreover, previous hospitalization for COPD exacerbation has been reported to be an important prognostic factor in patients with COPD exacerbation. ${ }^{4,5}$

Antibiotics are used mainly in patients with moderate or severe exacerbations and/or cough and sputum purulence. ${ }^{6} \mathrm{An}$ initial empirical treatment with narrow-spectrum antibiotics, such as aminopenicillin with clavulanic acid, macrolide, and tetracycline, is recommended for the entire population with COPD exacerbation, based on previous systematic reviews. ${ }^{7,8}$ However, evidence that can guide decisions regarding whether to choose narrow-spectrum or broad-spectrum antibiotics is lacking. Based on expert opinions, local bacterial resistance patterns should be considered. ${ }^{6}$ Furthermore, for patients with frequent exacerbations, anti-pseudomonal antibiotic use should be considered, as frequent COPD exacerbations have been reported as associated with the isolation of Pseudomonas aeruginosa (PA). ${ }^{9}$ However, it is unclear whether empirical treatment for PA can improve outcomes in patients with frequent COPD exacerbations. ${ }^{10}$ Therefore, we aimed to assess the effectiveness of empirical anti-pseudomonal antibiotics in patients with recurrent COPD exacerbation. We hypothesized that anti-pseudomonal antibiotics would improve the length of the hospital stay in patients with frequent COPD exacerbation.

\section{Methods}

\section{Study design}

The study was designed as a multicenter retrospective cohort study across seven acute general hospitals in Japan: Awa Regional Medical Center, Hyogo Prefectural Amagasaki General Medical Center, Ichinomiyanishi Hospital, Kameda Medical Center, Kobe City Medical Center General Hospital, Saiseikai Yokohamashi Tobu Hospital, and Tokyo National Hospital. This study was conducted in accordance with the Declaration of Helsinki. ${ }^{11}$ Additionally, this study was approved by the Institutional Review Board (IRB) of each hospital (registration number: 2020008), and the need for written informed consent was waived by the IRB of Ichinomiyanichi Hospital. This article was prepared in accordance with the Strengthening the Reporting of Observational Studies in Epidemiology (STROBE) Statement (e-Table 1). ${ }^{12}$

\section{Patient selection}

The patient enrollment period depended on the storage terms of the electronic medical records in each hospital during the study period of April 1, 2008 to July 31, 2020. Inclusion criteria were age $\geq 40$ years and hospital admittance due to COPD exacerbation $\geq 2$ times during the study period. Hospital admittance due to COPD exacerbation was determined by an admission-precipitating diagnosis of COPD exacerbation based on the 10th revision of the International Statistical Classification of Diseases and Related Health Problems (ICD-10) code (ICD-10 code: J44.1). The exclusion criteria were as follows: 
empirical use of anti-pseudomonal antibiotics for multidrug-resistant PA that are available in Japan (i.e. colistin, polymyxin B, and fosfomycin).

A validation study of the patient selection based on the ICD-10 code (J44.1) was conducted at Ichinomiyanishi Hospital, Kameda Medical Center, Saiseikai Yokohamashi Tobu Hospital, and Tokyo National Hospital during the study period. Patients who were selected according to the above inclusion criteria were reviewed using the clinical charts from a respiratory physician to confirm the diagnosis of COPD exacerbation.

\section{Data collection}

The following patient data were collected from the Diagnosis Procedure Combination database: age, admission date, discharge date, sex, height, weight, activities of daily living, comorbidities, tracheal intubation, and prognosis. Other patient data were collected through the review of electrical medical records: baseline COPD stage, type of inhaler used (inhaled corticosteroid, long-acting beta2-agonist, long-acting muscarinic antagonist), home oxygen therapy use, activities of daily living as assessed by the Barthel index, prior PA isolation, vital signs (systolic blood pressure, respiratory rate, heart rate), mental status, oxygen use on admission, systemic steroid therapy, and antibiotic therapy.

\section{Outcomes}

The primary outcome was the length of the hospital stay. The outcome was measured repeatedly for each patient, and observations were nested within individuals and hospitals. Thus, patients and hospitals were considered as clusters.

\section{Treatment of interest}

The treatment of interest comprised the empirical use of anti-pseudomonal antibiotics on admission or the next day, regardless of the dose and route of administration. Anti-pseudomonal antibiotics comprised drugs that are available in Japan, as follows: ceftazidime, cefozopran, cefepime, carbapenem (biapenem, doripenem, imipenem, and meropenem), piperacillin, piperacillin/tazobactam, aminoglycoside (gentamicin, tobramycin, and amikacin), quinolone (ciprofloxacin, levofloxacin, garenoxacin, and gatifloxacin), and aztreonam.

\section{Covariates}

Based on the previous literature, the following factors were selected as potential confounding factors: age, body mass index, home oxygen therapy use (binary data), respiratory rate, heart rate $(\geq 109$ beats/min or not), oxygen use on admission (binary data), mental status (altered mental status or not), systemic steroid use on admission or the next day regardless of the dose (binary data), activities of daily living (Barthel index), and number of recurrences. ${ }^{4,13-18}$

\section{Statistical analysis}


Patient characteristics are summarized as numbers and percentages for categorical variables and as the median and interquartile range (IQR) for continuous variables. A log-linked Gamma model was used to evaluate the association between each variable and the length of the hospital stay. ${ }^{19}$ Since repeated observations were obtained from a single patient, we used the generalized estimating equation method with an exchangeable correlation structure and robust standard error estimation. Hospital-specific effects were implemented as fixed effects.

Missing data were imputed using multiple imputations by chained equations on the assumption that data were missing at random. ${ }^{20}$ Covariates of the outcome analysis (number of recurrences, age, home oxygen therapy use, oxygen use on admission, heart rate, respiratory rate, mental status, systemic steroid use, admitting hospital), the treatment variable (anti-pseudomonal antibiotic use), and the outcome variable (length of the hospital stay), in addition to systolic blood pressure as an auxiliary variable, were used to estimate the missing data. ${ }^{21,22}$ The results for each imputed dataset were aggregated using Rubin's rule. ${ }^{23}$

We also constructed a Bayesian model in which both patient- and hospital-specific effects were implemented as random effects using the complete case dataset. ${ }^{24}$ This model can be described as follows:

$$
\begin{aligned}
& Y_{i j k} \sim \operatorname{Gamma}\left(s, s / \mu_{i j k}\right) \\
& \log \left(\mu_{i j k}\right)=X_{i j k} \beta+b_{j}+b_{k} \\
& \sum b_{j}=0, \sum b_{k}=0
\end{aligned}
$$

where $Y_{i j k}$ is the length of the $i$-th stay of patient $j$ in hospital $k$, and the mean of the Gamma distribution, $\log (\mu)$, is determined by explanatory variables, $X$, and the patient- and hospital-specific effects with a zerosum constraint, $\mathrm{b}_{j}$ and $\mathrm{b}_{k}$. We set four separate sampling chains, each consisting of 5000 samples (including 4000 samples discarded for convergence). We evaluated the sampling convergence using the Gelman-Rubin statistic (R-hat) and visually inspected the trace plot.

All statistical analyses were performed using R software version 4.0.2 (R Foundation for Statistical Computing, Vienna, Austria). For Bayesian model analyses, we used the probabilistic programming language, Stan (Stan Development Team). The script is available on GitHub (https://github.com/AkihiroShiroshita/Recurrent-COPD-AE). ${ }^{25}$

\section{Results}

The validation study conducted across four hospitals revealed that patient selection for recurrent COPD exacerbation using the ICD-10 code resulted in 6 of 108 false positives (positive predictive value of 94\%). 
The patient selection flowchart is shown in Figure 1. Among the selected 1574 patients with COPD exacerbation, 344 (22\%) were hospitalized with an admission-precipitating diagnosis of COPD exacerbation $\geq 2$ times during the study period (total number of observations/cases: 965 ). No patients were excluded because of the empirical use of anti-pseudomonal antibiotics for multidrug-resistant PA. The median number of recurrences was 3 (IQR: 2-4), and the median interval to the next hospitalization was 176 days (IQR: 55-507 days). The patients' characteristics are summarized according to treatment in Table 1.

PA was detected in at least 75 of 344 patients (22\%) during the study period. The strategies for empirical antibiotic use are summarized in Table 2 and e-Table 2. Piperacillin/tazobactam was used in $70 \%$ of antipseudomonal antibiotic cases, and ceftriaxone was used in $73 \%$ of non-anti-pseudomonal antibiotic cases. Although a sputum sample was not collected in all cases, at least 75 of 965 observations/cases (8\%) showed positive sputum-culture results for PA before admission (characterized in e-Table 3 ). Among these 75 cases, anti-pseudomonal antibiotics were used in 20 (27\%). None of the patients received double coverage with anti-pseudomonal antibiotics. Only $13 \%$ of cases received antibiotics covering atypical respiratory pathogens, including Mycoplasma pneumonia, Legionella spp., and Chlamydia spp. In the non-anti-pseudomonal antibiotics group, 18 of 474 cases (4\%) were switched to anti-pseudomonal antibiotics during hospitalization.

The main analysis with multiple imputations and the generalized estimating equation revealed that the estimated change in the length of the hospital stay between the anti-pseudomonal and non-antipseudomonal antibiotics groups was 0.044 days [95\% confidence interval; $-0.077,0.166$ ] (Table 3 ). This non-significant result was consistent with the results of the sensitivity analysis.

\section{Discussion}

This multicenter retrospective cohort study aimed to evaluate the effectiveness of empirical antipseudomonal antibiotics in terms of the length of the hospital stay in patients with frequent COPD exacerbation. We hypothesized that anti-pseudomonal antibiotics would reduce the length of the hospital stay. However, after adjusting for known confounding factors, the length of the hospital stay was not significantly different between the non-anti-pseudomonal and anti-pseudomonal antibiotic groups.

Although the PA detection rate in the present study was not lower than that in previous studies conducted in other countries ( $22 \%$ in the present study vs. $4 \%-13 \%$ ), our hypothesis was not supported. ${ }^{10,26}$ To the best of our knowledge, this study is the first to assess the effectiveness of anti-pseudomonal antibiotics in patients with recurrent COPD exacerbation. Our non-significant results are consistent with a previous prospective cohort study targeting hospitalized patients who had a positive culture result for PA in lowertract specimens. ${ }^{27}$ In this previous study, only $18 \%$ of patients were treated with anti-pseudomonal antibiotics and, after adjusting for confounding factors, inadequate initial antibiotic use was not associated with 12-month mortality. In contrast, inappropriate initial antibiotic use in the context of PA pneumonia has been reported as associated with increased 28 -day mortality. ${ }^{28}$ This difference in results 
may be due to differences in the proportion of patients with pneumonia. In a study of PA pneumonia, the 28-day mortality rate was $51 \%$, which is comparable to that in other reports. ${ }^{29}$ Although PA detection among patients with COPD has been reported as associated with extremely high 2-year all-cause mortality $(23 \%-41 \%)$, the 1 -month mortality was $0 \% .{ }^{10,30}$ Considering the low 1 -month mortality in patients with COPD exacerbation with PA isolation, empirical anti-pseudomonal antibiotics may not have adequate power to change the prognosis during hospitalization.

Initial treatment with non-anti-pseudomonal antibiotics may spare anti-pseudomonal antibiotic use. The median time to the next hospitalization was approximately half a year, and the previous use of antibiotics was detected in only one-third of observations. In the present study, although at least 70 observations/cases had positive sputum-culture results for PA before admission, PA was covered by empirical antibiotics in only $29 \%$ of cases. The patient characteristics and length of the hospital stay were not substantially different between anti-pseudomonal and non-anti-pseudomonal antibiotic groups. Furthermore, a change in a treatment from non-anti-pseudomonal antibiotics to anti-pseudomonal antibiotics occurred in only $4 \%$ of cases. Thus, physicians may not need to administer anti-pseudomonal antibiotics based only on the number of recurrences. Currently, multidrug-resistant organisms are a global concern. ${ }^{31}$ The judicious use of broad-spectrum antibiotics can reduce the number of drug-resistant microorganisms. ${ }^{32,33}$ Additionally, the use of broad-spectrum antibiotics is associated with a high cost of care and increased antibiotic-associated side effects, such as Clostridium difficile infection. ${ }^{34}$ Therefore, a strategy of empirical narrow-spectrum antibiotic use in patients with recurrent COPD exacerbation may be an acceptable choice.

This multicenter retrospective study has numerous strengths. First, this study was based on daily clinical practice. In contrast to the GOLD 2020 report and a network meta-analysis of randomized controlled trials (RCTs), the antibiotics used in the present cohort were mainly intravenous antibiotics. ${ }^{6,35}$ This may be due to the large number of elderly patients that required some assistance; the average age of the present cohort was 80 years, and only 175 of 965 (18\%) cases were fully independent. The present study results may better reflect hospitalized patients in daily practice rather than selected patients in RCTs. ${ }^{8}$ Second, the number of observations was large compared to that in previous RCTs. The number of included patients/observations was much larger than that in a meta-analysis of four RCTs assessing the effect of currently used antibiotics on the length of the hospital stay (965 observations in the present study vs. 393). ${ }^{8}$ Furthermore, an ongoing RCT targeting patients with at least one hospitalization within the prior 12 months and the detection of PA in a sputum culture has an expected total sample size of 150 (for the comparison of the anti-pseudomonal antibiotics group and the placebo group), ${ }^{36}$ which is far smaller than that in the present study. Thus, the present results may fill gaps not covered by these RCTs.

The main caveat regarding this study is that we only evaluated the length of the hospital stay. Although the length of the hospital stay is associated with the survival of hospitalized patients with COPD, as an outcome, it is short-term and soft. Based on the results of previous systematic reviews, hard outcomes such as in-hospital death and 30-day mortality could not be evaluated in the present study because of its 
sample size. ${ }^{7,8}$ Moreover, the time to the next exacerbation could not be evaluated because of unmeasured confounding factors, such as post-admission baseline treatments for COPD. In a previous retrospective cohort study with unmeasured confounding factors, antibiotic use was associated with improvements in the long-term mortality and time to the next exacerbation. ${ }^{37}$ Further large-scale studies are warranted to evaluate hard short-term and long-term outcomes. ${ }^{38}$ The present study had several other limitations. First, although our validation study showed a high predictive value for our patient selection strategy, the number of patients with COPD exacerbation was relatively small, considering that all of the hospitals were acute care and educational hospitals. Patient selection based on the ICD-10 code could have low sensitivity, and there could be many patients who should have been included in this study. In Japan, especially in our hospitals, payment was based on the Diagnosis Procedure Combination system, in which hospitals were reimbursed based on the diagnosis code of hospitalized patients. Patients with a long length of stay or complications during hospitalization might be coded as diagnoses other than COPD exacerbation. Thus, the length of stay may have been right-truncated, and the effect of antipseudomonal antibiotics may have been skewed toward the null. Second, the interval between COPD exacerbations was not taken into account, and the variance correlation of the length of the hospital stay within each patient over multiple visits may differ from our expectations. We coped with this issue by using robust variances. Third, there may be additional confounding factors. For example, because of the substantial amount of missing data regarding the COPD stage, we could not use it as a covariate; accordingly, the estimate of the effect of anti-pseudomonal antibiotics might have been skewed toward the null. Although we used other similar baseline covariates, such as the activities of daily living and home oxygen therapy use, an additional confounding factor may have altered the results. Further RCTs are needed to address unknown confounding factors.

\section{Interpretation}

In conclusion, the present study results suggest that empirical anti-pseudomonal antibiotics do not decrease the length of the hospital stay. Thus, empirical anti-pseudomonal antibiotic use based only on recurrence may not be necessary. However, further studies with larger sample sizes are needed to evaluate the effectiveness of anti-pseudomonal antibiotics more precisely.

\section{Abbreviations}

COPD, chronic obstructive pulmonary disease; ICD-10, 10th revision of the International Statistical Classification of Diseases and Related Health Problems; IQR, interquartile range; IRB, Institutional Review Board; PA, Pseudomonas aeruginosa; RCT, randomized controlled trial; STROBE, Strengthening the Reporting of Observational Studies in Epidemiology

\section{Declarations}

Guarantor: Dr. Yuki Kataoka takes responsibility for the content of the manuscript, including the data and analysis. 
Acknowledgments: The authors would like to thank Hiroyuki Suzuki at the Department of Infectious Diseases of lowa University in the United States for providing us with a suggestion on multidrug-resistant bacteria.

Conflict of interest: The authors declare no competing financial interests.

Funding source: Funding for English-language editing was obtained from the Systematic Review Workshop Peer Support Group (SRWS-PSG: https://community.camp-fire.jp/projects/view/187310). The funder played no role in the study design, study execution, data analyses, data interpretation, or decision to submit the results.

Author contributions: AS, ST, HS, CS, KS, SM, Yuya K, KT, and Yuki Kataoka contributed to the conception and design of the work. AS, ST, HS, CS, KS, SM, Yuya K, KT, and MO contributed to data acquisition. AS, $\mathrm{CM}$, and Yuki Kataoka contributed to the data analysis and interpretation. AS, CM, and Yuki Kataoka drafted the manuscript. All authors revised the manuscript critically and approved the final version of the manuscript. AS, CM, ST, HS, CS, KS, SM, Yuya K, KT, MO, and Yuki K agreed to be accountable for all aspects of any part of the work.

\section{References}

1. Halbert RJ, Isonaka S, George D, Iqbal A. Interpreting COPD prevalence estimates: what is the true burden of disease? Chest. 2003;123(5):1684-1692.

2. Hurst JR, Vestbo J, Anzueto A, et al. Susceptibility to exacerbation in chronic obstructive pulmonary disease. N Engl J Med. 2010;363(12):1128-1138.

3. Cote CG, Dordelly LJ, Celli BR. Impact of COPD exacerbations on patient-centered outcomes. Chest. 2007;131(3):696-704.

4. Echevarria C, Steer J, Heslop-Marshall K, et al. The PEARL score predicts 90-day readmission or death after hospitalisation for acute exacerbation of COPD. Thorax. 2017;72(8):686-693.

5. Matkovic Z, Huerta A, Soler N, et al. Predictors of adverse outcome in patients hospitalised for exacerbation of chronic obstructive pulmonary disease. Respiration. 2012;84(1):17-26.

6. 2020 Gold Reports. Global initiative for chronic obstructive lung disease - GOLD. 2020. https://goldcopd.org/gold-reports. Accessed 5 Mar 2021.

7. Dobler CC, Morrow AS, Beuschel B, et al. Pharmacologic therapies in patients with exacerbation of chronic obstructive pulmonary disease: a systematic review with meta-analysis. Ann Intern Med. 2020;172(6):413-422.

8. Vollenweider DJ, Frei A, Steurer-Stey CA, Garcia-Aymerich J, Puhan MA. Antibiotics for exacerbations of chronic obstructive pulmonary disease. Cochrane Database Syst Rev. 2018;10(10):CD010257.

9. Garcia-Vidal C, Almagro P, Romaní V, et al. Pseudomonas aeruginosa in patients hospitalised for COPD exacerbation: a prospective study. Eur Respir J. 2009;34(5):1072-1078. 
10. Eklöf J, Sørensen R, Ingebrigtsen TS, et al. Pseudomonas aeruginosa and risk of death and exacerbations in patients with chronic obstructive pulmonary disease: an observational cohort study of 22053 patients. Clin Microbiol Infect. 2020;26(2):227-234.

11. World Medical Association. WMA Declaration of Helsinki - Ethical Principles for Medical Research Involving Human Subjects. 2018. https://www.wma.net/policies-post/wma-declaration-of-helsinkiethical-principles-for-medical-research-involving-human-subjects. Accessed 11 Mar 2021.

12. von Elm E, Altman DG, Egger M, et al. The Strengthening the Reporting of Observational Studies in Epidemiology (STROBE) statement: guidelines for reporting observational studies. Ann Intern Med. 2007;147(8):573-577.

13. Tabak YP, Sun X, Johannes RS, Gupta V, Shorr AF. Mortality and need for mechanical ventilation in acute exacerbations of chronic obstructive pulmonary disease: development and validation of a simple risk score. Arch Intern Med. 2009;169(17):1595-1602.

14. Wilson DO, Rogers RM, Wright EC, Anthonisen NR. Body weight in chronic obstructive pulmonary disease. The National Institutes of Health Intermittent Positive-Pressure Breathing Trial. Am Rev Respir Dis. 1989;139(6):1435-1438.

15. Schols AM, Slangen J, Volovics L, Wouters EF. Weight loss is a reversible factor in the prognosis of chronic obstructive pulmonary disease. Am J Respir Crit Care Med. 1998;157(6 Pt 1):1791-1797.

16. Quintana JM, Esteban C, Unzurrunzaga A, et al. Predictive score for mortality in patients with COPD exacerbations attending hospital emergency departments. BMC Med. 2014;12:66.

17. Flattet $Y$, Garin N, Serratrice J, Perrier A, Stirnemann J, Carballo S. Determining prognosis in acute exacerbation of COPD Int J Chron Obstruct Pulmon Dis. 2017;12:467-475.

18. Walters JA, Tan DJ, White CJ, Gibson PG, Wood-Baker R, Walters EH. Systemic corticosteroids for acute exacerbations of chronic obstructive pulmonary disease. Cochrane Database Syst Rev. 2014; (9):CD001288.

19. Williford E, Haley V, McNutt LA, Lazariu V. Dealing with highly skewed hospital length of stay distributions: The use of Gamma mixture models to study delivery hospitalizations. PLoS One. 2020;15(4):e0231825.

20. White IR, Royston P, Wood AM. Multiple imputation using chained equations: Issues and guidance for practice. Stat Med. 2011;30(4):377-399.

21. He Y. Missing data analysis using multiple imputation: getting to the heart of the matter. Circ Cardiovasc Qual Outcomes. 2010;3(1):98.

22. Moons KG, Donders RA, Stijnen T, Harrell FE Jr. Using the outcome for imputation of missing predictor values was preferred. J Clin Epidemiol. 2006;59(10):1092-1101.

23. Marshall A, Altman DG, Holder RL, Royston P. Combining estimates of interest in prognostic modelling studies after multiple imputation: current practice and guidelines. BMC Med Res Methodol. 2009;9:57.

24. Manandhar B, Nandram B. Hierarchical Bayesian models for continuous and positively skewed data from small areas. Communications in Statistics - Theory and Methods. 2021;50(4):944-962. 
25. R: The R Project for Statistical Computing. https://www.r-project.org. Accessed 18 Feb 2021.

26. Lieberman D, Lieberman D. Pseudomonal infections in patients with COPD: epidemiology and management. Am J Respir Med. 2003;2(6):459-468.

27. Eklöf J, Gliese KM, Ingebrigtsen TS, Bodtger U, Jensen JS. Antibiotic treatment adequacy and death among patients with Pseudomonas aeruginosa airway infection. PLoS One. 2019;14(12):e0226935.

28. Park SY, Park HJ, Moon SM, et al. Impact of adequate empirical combination therapy on mortality from bacteremic Pseudomonas aeruginosa pneumonia. BMC Infect Dis. 2012;12:308.

29. Fujitani S, Sun HY, Yu VL, Weingarten JA. Pneumonia due to Pseudomonas aeruginosa: Part l: epidemiology, clinical diagnosis, and source. Chest. 2011;139(4):909-919.

30. Rodrigo-Troyano A, Melo V, Marcos PJ, et al. Pseudomonas aeruginosa in chronic obstructive pulmonary disease patients with frequent hospitalized exacerbations: a prospective multicentre study. Respiration. 2018;96(5):417-424.

31. World Health Organization. Antimicrobial resistance. https://www.who.int/news-room/factsheets/detail/antimicrobial-resistance. 2020. Accessed 27 Mar 2021.

32. Lewis GJ, Fang X, Gooch M, Cook PP. Decreased resistance of Pseudomonas aeruginosa with restriction of ciprofloxacin in a large teaching hospital's intensive care and intermediate care units. Infect Control Hosp Epidemiol. 2012;33(4):368-373.

33. Stultz JS, Arnold SR, Shelton CM, Bagga B, Lee KR. Antimicrobial stewardship impact on Pseudomonas aeruginosa susceptibility to meropenem at a tertiary pediatric institution. Am J Infect Control. 2019;47(12):1513-1515.

34. Webb BJ, Sorensen J, Jephson A, Mecham I, Dean NC. Broad-spectrum antibiotic use and poor outcomes in community-onset pneumonia: a cohort study. Eur Respir J. 2019;54(1):1900057.

35. Zhang HL, Tan M, Qiu AM, Tao Z, Wang CH. Antibiotics for treatment of acute exacerbation of chronic obstructive pulmonary disease: a network meta-analysis. BMC Pulm Med. 2017;17(1):196.

36. Eklöf J. Targeted AntiBiotics for Chronic Obstructive Pulmonary Disease (Target-ABC). 2020. https://clinicaltrials.gov/ct2/show/NCT03262142. Accessed 4 Mar 2021.

37. Roede BM, Bresser P, Prins JM, Schellevis F, Verheij TJ, Bindels PJ. Reduced risk of next exacerbation and mortality associated with antibiotic use in COPD. Eur Respir J. 2009;33(2):282-288.

38. Gunen $\mathrm{H}$, Hacievliyagil SS, Kosar F, et al. Factors affecting survival of hospitalised patients with COPD. Eur Respir J. 2005;26(2):234-241.

\section{Tables}

Table 1. Patient characteristics per observation 


\begin{tabular}{|c|c|c|c|}
\hline Characteristic & $\begin{array}{l}\text { Non-anti- } \\
\text { pseudomonal } \\
\text { antibiotics group } \\
\left(\mathrm{N}^{\star}=792,82 \%\right)\end{array}$ & $\begin{array}{l}\text { Anti- } \\
\text { pseudomonal } \\
\text { antibiotics } \\
\text { group } \\
(\mathrm{N}=173,18 \%)\end{array}$ & $\begin{array}{l}\text { Total } \\
(\mathrm{N}=965)\end{array}$ \\
\hline \multicolumn{4}{|l|}{ Demographic characteristics } \\
\hline Age (years, mean, $\mathrm{SD}^{\dagger}$ ) & $78(9)$ & $80(8)$ & $78(9)$ \\
\hline Male (number, \%) & $697(88)$ & $154(89)$ & $851(88)$ \\
\hline Height (cm, SD) & $160(11)$ & $161(8)$ & $160(10)$ \\
\hline Missing data (number, \%) & $156(20)$ & $32(19)$ & $187(20)$ \\
\hline Weight $(\mathrm{kg}, \mathrm{SD})$ & $53(12)$ & $52(12)$ & $52(12)$ \\
\hline Missing data (number, \%) & $133(17)$ & $11(6)$ & $143(15)$ \\
\hline \multicolumn{4}{|l|}{ COPD ${ }^{\ddagger}$ Stage } \\
\hline$\nabla($ number, \%) & $36(9)$ & $2(4)$ & $38(9)$ \\
\hline$\nabla($ number, \%) & $120(31)$ & $18(40)$ & $138(32)$ \\
\hline$\nabla($ number, \%) & $155(40)$ & $15(33)$ & $170(39)$ \\
\hline$\nabla($ number, \%) & $79(20)$ & $10(22)$ & $89(21)$ \\
\hline Missing data (number, \%) & $402(51)$ & $128(74)$ & $530(55)$ \\
\hline Inhaled corticosteroid (number, \%) & $288(36)$ & $78(45)$ & $366(38)$ \\
\hline Long-acting beta2-agonist (number, \%) & $427(54)$ & $109(63)$ & $536(56)$ \\
\hline $\begin{array}{l}\text { Long-acting muscarinic antagonist } \\
\text { (number, \%) }\end{array}$ & $493(62)$ & $114(66)$ & $607(63)$ \\
\hline Home oxygen therapy users (number, \%) & $347(44)$ & $66(38)$ & $413(43)$ \\
\hline $\begin{array}{l}\text { Activities of daily living score" (score, } \\
\text { IQR§) }\end{array}$ & $45[10-80]$ & $30[0-55]$ & $45[5-70]$ \\
\hline Missing data (number, \%) & $128(16)$ & $25(15)$ & $13(1)$ \\
\hline $\begin{array}{l}\text { Median number of recurrences (number, } \\
\text { IQR) }\end{array}$ & $3[3-4]$ & $3[3-5]$ & $3[2-4]$ \\
\hline $\begin{array}{l}\text { Median time to next hospitalization (days, } \\
\text { IQR) }\end{array}$ & $183[58-533]$ & $151[53-424]$ & $\begin{array}{l}176[55- \\
507]\end{array}$ \\
\hline \multicolumn{4}{|l|}{ Comorbidities } \\
\hline Asthma (number, \%) & $149(19)$ & $39(23)$ & $188(20)$ \\
\hline
\end{tabular}




\begin{tabular}{|c|c|c|c|}
\hline Heart failure (number, \%) & $142(18)$ & $31(18)$ & $173(18)$ \\
\hline Cancer (number, \%) & $83(11)$ & $16(9)$ & $99(10)$ \\
\hline Diabetes mellitus (number, \%) & $107(14)$ & $35(20)$ & $142(15)$ \\
\hline \multicolumn{4}{|l|}{ Vital signs } \\
\hline Altered mental status (number, \%) & $105(13)$ & $29(17)$ & $134(14)$ \\
\hline $\begin{array}{l}\text { Systolic blood pressure (mmHg, mean, } \\
\text { SD) }\end{array}$ & $138(27)$ & $133(27)$ & $137(27)$ \\
\hline Missing data (number, \%) & $41(5)$ & $2(1)$ & $43(4)$ \\
\hline Heart rate (beats/min, mean, SD) & $99(20)$ & $105(22)$ & $100(20)$ \\
\hline Missing data & $30(4)$ & $4(2)$ & $34(4)$ \\
\hline Respiratory rate (breaths/min, mean, SD) & $24(6)$ & $26(7)$ & $25(6)$ \\
\hline Missing data (number, \%) & $62(8)$ & $18(10)$ & $80(8)$ \\
\hline Oxygen use on admission (number, \%) & $604(76)$ & $140(81)$ & $744(77)$ \\
\hline Missing data (number, \%) & $19(2)$ & $0(0)$ & $19(2)$ \\
\hline \multicolumn{4}{|l|}{ Treatment } \\
\hline Steroid therapy (number, \%) & $663(84)$ & $160(93)$ & $823(85)$ \\
\hline \multicolumn{4}{|l|}{ Prognosis } \\
\hline Length of hospital stay (days, IQR) & $11[8-17]$ & $12[9-21]$ & $12[8-18]$ \\
\hline Tracheal intubation (number, \%) & $39(5)$ & $17(10)$ & $56(6)$ \\
\hline Death (number, \%) & $37(5)$ & $18(10)$ & $55(6)$ \\
\hline
\end{tabular}

Abbreviations: *: $\mathrm{N}=$ number; $\mathrm{\dagger}: \mathrm{SD}=$ standard deviation; $\neq:$ COPD = chronic obstructive pulmonary disease; $\S \mathrm{IQR}$ = interquartile range

9: Activities of daily living score is defined as the Barthel index. A high score indicates a higher activity level.

Table 2. Empirical antibiotics therapy 


\begin{tabular}{|c|c|}
\hline Antibiotics & Number \\
\hline \multicolumn{2}{|c|}{ Anti-pseudomonal antibiotics $(\mathrm{N}=173)$} \\
\hline Ceftazidime & 7 \\
\hline Cefozopran & 2 \\
\hline Cefepime & 16 \\
\hline Carbapenem & 6 \\
\hline Biapenem & 0 \\
\hline Doripenem & 0 \\
\hline Imipenem & 0 \\
\hline Meropenem & 6 \\
\hline Piperacillin & 4 \\
\hline Piperacillin/tazobactam & 120 \\
\hline Aminoglycoside & 1 \\
\hline Gentamicin & 0 \\
\hline Tobramycin & 0 \\
\hline Amikacin & 1 \\
\hline Quinolone & 13 \\
\hline Ciprofloxacin & 1 \\
\hline Levofloxacin & 11 \\
\hline Garenoxacin & 1 \\
\hline Gatifloxacin & 0 \\
\hline Aztreonam & 4 \\
\hline \multicolumn{2}{|c|}{ Non-anti-pseudomonal antibiotics $(\mathrm{N}=474)$} \\
\hline Amoxicillin & 5 \\
\hline Amoxicillin/clavulanic acid & 22 \\
\hline Ampicillin & 4 \\
\hline Cefuroxime & 1 \\
\hline Penicillin-G & 2 \\
\hline Sulbactam/ampicillin & 56 \\
\hline
\end{tabular}

Page 15/17 


\begin{tabular}{|ll|} 
Cefotiam & 4 \\
\hline Ceftriaxone & 2 \\
\hline Vancomycin & 345 \\
\hline Sulfamethoxazole/trimethoprim & 4 \\
\hline Macrolide & 64 \\
\hline Azithromycin & 46 \\
\hline Clarithromycin & 1 \\
\hline Erythromycin & 17 \\
\hline Tetracycline & 7 \\
\hline Minomycin & 6 \\
\hline Doxycycline & 1 \\
\hline Moxifloxacin & 1 \\
\hline
\end{tabular}

Table 3. The estimated difference in the length of the hospital stay between the anti-pseudomonal and non-anti-pseudomonal antibiotics groups

\begin{tabular}{|lll|}
\hline Statistical model & Coefficient & $\begin{array}{l}95 \% \text { confidence interval } \\
\text { or 95\% highest density } \\
\text { interval }\end{array}$ \\
\hline $\begin{array}{l}\text { Multiple imputations and the generalized estimating } \\
\text { equation with an exchangeable correlation structure }\end{array}$ & 0.044 & $-0.077,0.166$ \\
$\begin{array}{l}\text { A complete case analysis and three-level Bayesian } \\
\text { hierarchical model }\end{array}$ & 0.178 & $-1.836,2.061$ \\
\hline
\end{tabular}

\section{Figures}


COPD exacerbation: $\mathrm{N}=1574$

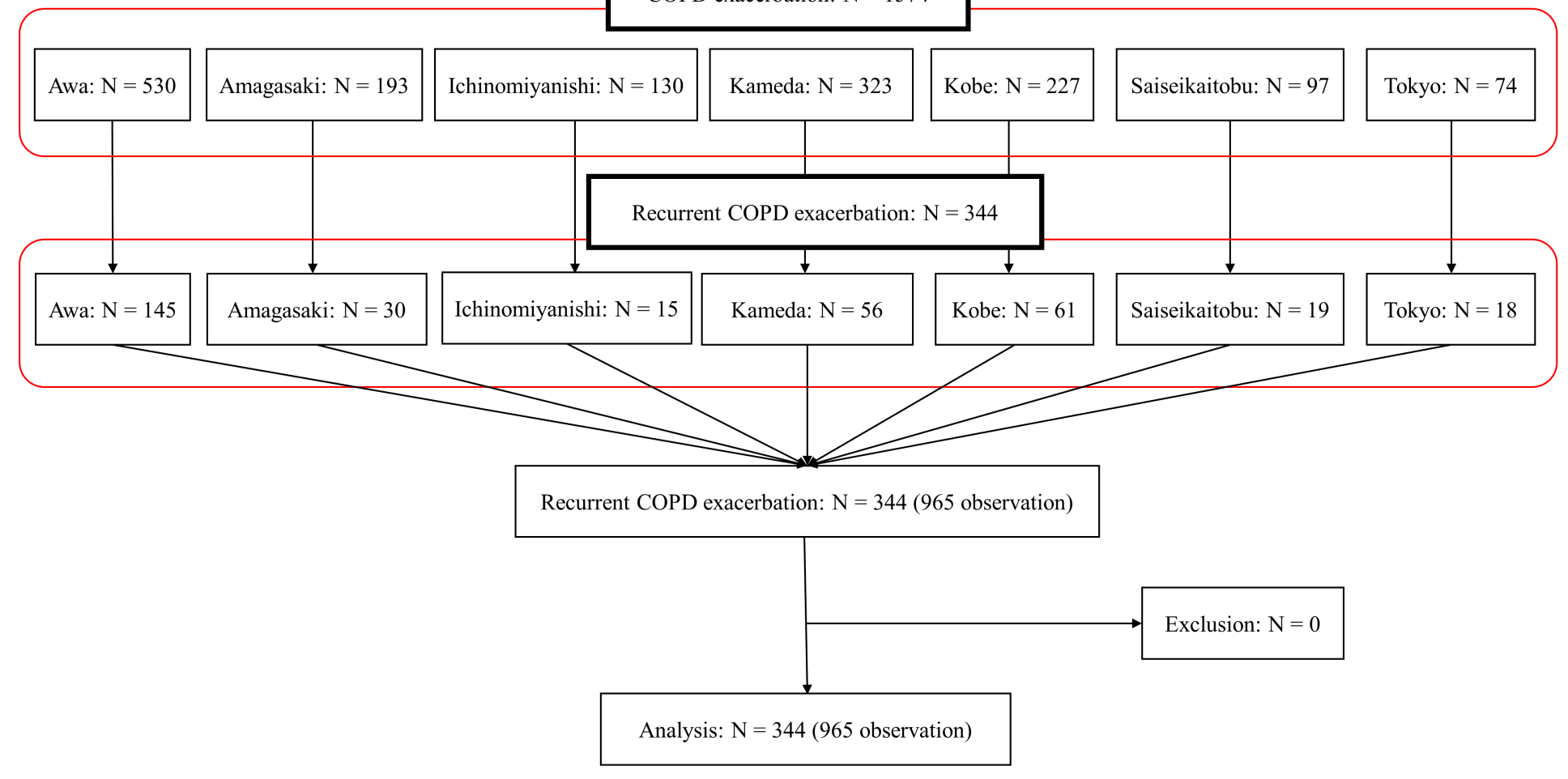

Figure 1

Patient selection flowchart. COPD = chronic obstructive pulmonary disease

\section{Supplementary Files}

This is a list of supplementary files associated with this preprint. Click to download.

- COPDsupplementarymaterialchest.docx 